\title{
PEDIATRICS
}

Chaychenko T., Rybka O., Buginskaya N.

\section{EXERCISE TOLERANCE IN NORMAL WEIGHT, UN- DERWEIGHT, OVERWEIGHT AND OBESE ADOLESCENTS}

Kharkiv National Medical University, Ukraine

\begin{abstract}
Lack of physical activity negatively impacts weight management programs effectiveness, even with the strictest dietary recommendations. In the context of the obesity epidemic, associated with the cardiovascular risk development, assessment of exercise tolerance in adolescents with different body composition assumes special significance. The examination of 64 normal weight, underweight, overweight and obese adolescents has been performed with anthropometric investigation, study of physical activity level by NHANES and exercise tolerance by multistage treadmill protocol. We established that normal weight children tolerate exercise better than underweight and overweight despite of the same physical activity level. Exercising for persons involved in of body mass correction programs must be adjusted to the potential cardiovascular complications, last longer with the less load in the boost and must be conducted under the relevant specialist control.
\end{abstract}

KeyWords: adolescents, body mass index, physical activity, exercise tolerance

\section{INTRODUCTION}

The WHO Global Strategy on Ending Childhood Obesity $(\mathrm{ECHO})$ includes a complex of measures at various levels from individual to socio-political one. Physical activity in this strategy plays a key role as the only way of energy expenditures [1]. Physical activity level in children is inversely proportional to the metabolic status [2]. Conversely, an adequate fitness reduces the cardiometabolic risk [3]. Obesity related cardiovascular disorders (hypertension, myocardial hypertrophy, remodeling) significantly limit the intensity of physical activity [4]. That's why a comparative analysis of exercise tolerance in children with different body composition it is necessary.

\section{Corresponding Author:}

Tetyana Chaychenko, MD, PhD, Professor of Department of Pediatrics No. 1 and Neonatology, Kharkiv National Medical University, Ukraine. E-mail: tatyana.chaychenko@gmail.com

\section{PURPOSES, SUBJECTS AND METHODS:}

\subsection{Purpose}

The aim of the study was to improve effectiveness and safety of weight management programs in adolescents by assessment of exercise tolerance in children with different body mass.

\subsection{Subjects \& Methods}

64 normal weight, underweight, overweight and obese (mean age 13,56+2,47 years) were examined. Grouping was done by the body mass index (BMI) Z-score: gr.S (skinny, underweight with $B M I$ less than $-1,0 S D, n=6$ ), gr.0 (normal weight with $B M I+1,0 S D, n=12$ ), gr.1 (overweight with $B M I$ $+1,1-2,0$ SD, $n=14$ ), gr.2 (obese with $B M I+2,1-3,0 S D$, $n=18$ ), gr.3 (obese with BMl more than $+3,0 S D, n=14$ ). Anthropometric examination included measurement of height, body mass, waist circumference and skin folds in standard positions. Abdominal fat predisposition assessed by the waist to height ratio (WHR) [5]. Body fat and lean body mass were calculated [6]. The physical activity readyness (PAR) assessed on the recommendations of NHANES, 2014 [7]. Multistage treadmill protocol (Bruce) 
used for exercise tolerance assessment with further analysis of cardiovascular parameters: resting heart rate $(\mathrm{HRr})$, maximal heart rate $(\mathrm{HRm})$, resting and maximal systolic and diastolic blood pressure (SBPr, SBPm, DBPr, DBPm respectively). Maximal predicted heart rate (MPHR) was calculated by Tanaka formula and $\mathrm{HRm}$ in patient was compared with MPHR as a percent of it (\%MPHR) [8]. Oxygen consumption calculated by ACSM formula to study cardiorespiratory fitness level [9].

The results were analyzed using Stat Soft Statistica 10. Quantitative variables were described as means $\pm \mathrm{SD}$, qualitative variables were described as percentages. Differences between groups were established by ANOVA and Mann-Whitney $U$ test. Reported P-values are two-tailed and $\mathrm{P}$-values <0,05 were considered to be statistically significant.

\section{Conflict of interests}

There is no conflict of interests.

\section{RESULTS AND DISCUSSION}

There was no gender and age difference between groups $(p>0,05)$ while BMI was gradually growing together with abdominal adiposity and sum of skinfolds (and body fat relevantly). Lean body mass in underweight is less comparatively to normal weight. It could be indirect confirmation that underweight are not athletes (Table 1).

There was no difference in basic cardiovascular parameters in groups (SBP, DBP, and HR). SBP and DBP were similar in skinny, normal weight and overweight, but high in all obese subjects. Resting HR did not reveal any difference in groups. Anyway, there was no significant difference in groups between maximal predicted heart rate and chronotropic reserve.

The physical activity level was significantly reduced only in the gr. 3 and different in others (where, regardless of BMI, children reported that were moderately active more than 1 hour per day). Normal weight children reached the maximal speed $(106.22+22.55 \mathrm{~m} / \mathrm{s})$ during exercise load, while the results of overweight and underweight were compared to each other. The lowest speed of movement as well as the smallest incline $(10.23+$ 5.41\%) were registered in children with the highest BMI.

Maximal oxygen consumption was also highest in normal weights and decreased in underweight and overweight with minimal result in heaviest ones. Maximal oxygen consumption to body mass gradually decreasing from group to group. At the same time, oxygen consumption referred to the lean body mass is same at. gr.S, gr.0 and gr. 1 but it is reduced compared to them in obese. Moreover, in obese with a $\mathrm{BMI}+>3 \mathrm{SD}$ the named parameter is twice lower than in those with a BMI + 2-3 SD. Thus, oxygen consumption (as a percentage of the predicted value) is identified a progressive decreasing from group to group. However, value is statistically reduced in obese and underweight.

The respiratory parameters (by the peak expiratory flow , PEF) were not changed in groups as well as oxygen saturation before, during and after the load.

The total distance passed during exercise boost was the longest in normal weight, gradually decreased in excess body mass and statistically lower in underweight. The same about exercise duration in groups.

Metabolic equivalent of exercising is decreasing while growing BMI. This means that at the same physical load in obese causes fewer calories burn out comparatively to underweight, normal weight and overweight.

Summarizing the data we can conclude, that normal weight children tolerate exercise better than underweight and overweight, physical activity for persons involved in of body mass correction programs must be adjusted to the potential cardiovascular complications, last longer with the less load in the boost and must be conducted under the relevant specialist control.

\section{CONCLUSIONS}

1. Fitness is reduced in both excess and deficiency of body weight despite of the same physical activity level. This is reflected by passage of the smaller distance with less tolerance to slope of surface and training time. 
Table 1.

Basic anthropometric parameters of groups and exercise tolerance markers in adolescents with different body mass

\begin{tabular}{|c|c|c|c|c|c|c|c|c|c|c|c|}
\hline \multirow{2}{*}{ Parameter } & \multicolumn{2}{|c|}{ Gr.S } & \multicolumn{2}{|c|}{ Gr.0 } & \multicolumn{2}{|c|}{ Gr.1 } & \multicolumn{2}{|c|}{ Gr.2 } & \multicolumn{2}{|c|}{ Gr.3 } & \multirow{2}{*}{$P$} \\
\hline & Mean & SD & Mean & SD & Mean & SD & Mean & SD & Mean & SD & \\
\hline \multicolumn{12}{|c|}{ Basic anthropometric parameters } \\
\hline $\mathrm{Z}-\mathrm{BMI}$ & $-1,61$ & 0,46 & $-0,20$ & 0,29 & 1,47 & 0,29 & 2,60 & 0,24 & 3,38 & 0,26 & $\mathrm{~S} 0, \mathrm{~S} 1, \mathrm{~S} 2, \mathrm{~S} 3,1-2,23,13$ \\
\hline$Z$ - height & $-0,78$ & 1,22 & $-0,29$ & 1,19 & 0,95 & 2,00 & 0,64 & 0,91 & 0,54 & 0,93 & \\
\hline WHR & 0,34 & 0,18 & 0,35 & 0,05 & 0,53 & 0,05 & 0,56 & 0,03 & 0,68 & 0,15 & $\mathrm{~S} 1, \mathrm{~S} 2, \mathrm{~S} 3,1-2,23,13$ \\
\hline Skin fold, $\mathrm{cm}$ & 21,33 & 29,57 & 41,18 & 42,29 & 118,29 & 42,29 & 154,28 & 33,02 & 181,62 & 28,94 & S0, S1, S2, S3, 1-2, 23, 13 \\
\hline Fat, \% & 19,64 & 5,80 & 27,04 & 4,49 & 37,97 & 4,49 & 41,75 & 2,89 & 43,93 & 2,41 & $\mathrm{~S} 0, \mathrm{~S} 1, \mathrm{~S} 2, \mathrm{~S} 3,1-2,23,13$ \\
\hline Lean mass, $\mathrm{kg}$ & 29,46 & 8,98 & 40,90 & 12,54 & 37,50 & 12,54 & 48,91 & 10,69 & 58,89 & 11,21 & S0, S1, S2, S3, 1-2, 23, 13 \\
\hline \multicolumn{12}{|c|}{ Exercise tolerance parameters } \\
\hline $\mathrm{SBPr}, \mathrm{mm} \mathrm{Hg}$ & 101,83 & 4,49 & 109,09 & 10,44 & 110,71 & 13,34 & 114,33 & 13,50 & 117,46 & 18,03 & \\
\hline DBPr, mm Hg & 63,33 & 6,06 & 70,45 & 8,50 & 69,71 & 8,65 & 75,28 & 11,65 & 76,85 & 12,11 & \\
\hline $\mathrm{HRr}, \mathrm{mm} \mathrm{Hg}$ & 70,50 & 6,02 & 74,64 & 10,50 & 77,64 & 12,70 & 79,67 & 11,58 & 84,46 & 11,87 & \\
\hline $\mathrm{SBPm}, \mathrm{mm} \mathrm{Hg}$ & 120,17 & 7,63 & 127,00 & 12,73 & 132,79 & 16,00 & 150,06 & 24,11 & 153,46 & 33,00 & $\mathrm{~S} 1, \mathrm{~S} 2, \mathrm{~S} 3,02,03,12$ \\
\hline $\mathrm{DBPm}, \mathrm{mm} \mathrm{Hg}$ & 72,50 & 7,58 & 80,00 & 8,94 & 80,71 & 12,06 & 90,28 & 10,02 & 93,85 & 21,83 & $\mathrm{~S} 1, \mathrm{~S} 2, \mathrm{~S} 3,02,03,12$ \\
\hline $\mathrm{HRm}, \mathrm{mm} \mathrm{Hg}$ & 142,17 & 18,71 & 135,55 & 25,35 & 127,86 & 32,09 & 116,89 & 27,85 & 129,46 & 27,34 & \\
\hline MPH, bpm & 199,95 & 2,41 & 197,69 & 1,26 & 199,00 & 1,90 & 198,36 & 1,88 & 198,25 & 1,63 & \\
\hline$\% \mathrm{MPH}$ & 71,08 & 9,20 & 68,52 & 12,53 & 64,32 & 16,47 & 58,92 & 14,05 & 65,34 & 14,05 & \\
\hline Chronotropic index & 29,12 & 0,00 & 40,94 & 0,00 & 36,36 & 23,70 & 25,26 & 26,80 & 38,69 & 29,61 & \\
\hline PAR & 3,33 & 1,86 & 2,36 & 1,69 & 3,79 & 1,76 & 3,11 & 1,28 & 1,62 & 1,39 & $13,23, \mathrm{~S} 3$ \\
\hline $\mathrm{V} \max , \mathrm{m} / \mathrm{sec}$ & 79,51 & 19,23 & 106,23 & 22,56 & 85,57 & 21,96 & 91,56 & 21,16 & 65,97 & 21,46 & $\mathrm{~S} 0, \mathrm{~S} 1, \mathrm{~S} 2, \mathrm{~S} 3,12,23,13$ \\
\hline Incline $\max , \%$ & 14,33 & 2,66 & 15,27 & 1,62 & 14,43 & 2,24 & 14,78 & 2,07 & 10,23 & 5,42 & $\mathrm{~S} 3,23,13$ \\
\hline $\mathrm{VO} 2 \mathrm{max}, \mathrm{ml} / \mathrm{min} / \mathrm{kg}$ & 29,96 & 7,97 & 39,60 & 8,62 & 32,04 & 8,62 & 34,30 & 8,33 & 22,57 & 10,45 & S0, S1, S2, S3, 12, 23, 13 \\
\hline METmax, kcal/min & 12,33 & 4,00 & 16,27 & 3,76 & 10,98 & 3,34 & 11,71 & 3,42 & 7,46 & 3,63 & S0, S1, S2, S3, 12, 23, 13 \\
\hline $\mathrm{VO} 2 \mathrm{l} / \mathrm{min}$ & 2,47 & 0,80 & 3,25 & 0,75 & 2,20 & 0,67 & 2,37 & 0,68 & 1,53 & 0,77 & \\
\hline Duration, min & 12,33 & 2,80 & 16,73 & 3,44 & 13,71 & 2,95 & 13,69 & 2,70 & 9,85 & 4,58 & S0, S1, S2, S3, 12, 23, 13 \\
\hline Distance, $\mathrm{m}$ & 545,00 & 135,90 & 990,00 & 291,38 & 657,14 & 230,70 & 745,00 & 254,03 & 469,23 & 286,75 & S0, S1, S2, S3, 12, 23, 13 \\
\hline $\mathrm{VO} 2,1 / \mathrm{min} / \mathrm{kg}$ & 0,08 & 0,04 & 0,07 & 0,03 & 0,04 & 0,02 & 0,03 & 0,01 & 0,02 & 0,01 & $\begin{array}{l}01,12,23,13,02,03, \mathrm{~S} 1, \\
\text { S2, S3 }\end{array}$ \\
\hline $\mathrm{VO} 2,1 / \mathrm{min} / \mathrm{kg}$ of lean & 0,08 & 0,04 & 0,08 & 0,04 & 0,06 & 0,03 & 0,05 & 0,02 & 0,03 & 0,02 & $13,23,02,03, \mathrm{~S} 2, \mathrm{~S} 3$ \\
\hline Observed VO2max, $\mathrm{ml} / \mathrm{min} / \mathrm{kg}$ & 79,77 & 40,36 & 66,33 & 29,97 & 39,39 & 17,39 & 28,70 & 11,82 & 15,44 & 10,20 & $\begin{array}{l}01,12,23,13,02,03, \mathrm{~S} 1, \\
\text { S2, S3 }\end{array}$ \\
\hline Predicted VO2 max, $\mathrm{ml} / \mathrm{min} / \mathrm{kg}$ & 45,52 & 4,98 & 43,00 & 4,18 & 44,28 & 6,56 & 40,58 & 5,96 & 34,58 & 4,83 & $23,13,03, \mathrm{~S} 3$ \\
\hline$\%$ of Predicted VO2 max & 66,17 & 17,64 & 92,56 & 21,01 & 74,08 & 24,14 & 85,42 & 20,91 & 64,25 & 27,75 & S0, 23,03, S3 \\
\hline PEF0, 1/min & 441,67 & 73,60 & 485,45 & 96,06 & 412,86 & 96,51 & 407,22 & 121,26 & 386,92 & 139,55 & \\
\hline PEFmax, $1 /$ min & 458,33 & 106,85 & 495,45 & 121,36 & 400,71 & 111,32 & 430,00 & 146,69 & 392,31 & 139,71 & \\
\hline $\mathrm{Sa} \mathrm{O} 2, \%$ & 98,33 & 11,34 & 98,80 & 3,79 & 100,60 & 2,99 & 99,14 & 2,85 & 99,33 & 1,97 & \\
\hline
\end{tabular}


2. In terms of cardiorespiratory fitness, the most unfavorable is the reduction of oxygen consumption, as normalized to total body weight so to the lean mass. Obese children are prone to hypertension after the exercise boost and, relevantly to the acute events.

3. Than heavier child than fewer calories burn out could be triggered by the same physical load, which should be taken into account when forecasting effective weight loss under the influence of exercising.

\section{REFERENCES}

1. Interim report of the Commission on Ending Childhood Obesity. World Health Organization 2015. Available from URL: http://www.who.int/end-childhoodobesity/commission-ending-childhood-obesity-interimreport.pdf?ua=1.

2. Parrett, A.L., Valentine, R.J., Arngrímsson, S.A., Castelli, D.M., Evans, E.M. (2011). Adiposity and aerobic fitness are associated with metabolic disease risk in children. ApplPhysiolNutrMetab, 36(1):72-9. doi: 10.1139/H10-083.

3. Brouwer, S.I., Stolk, R.P., Liem, E.T., Lemmink, K.A., Corpeleijn, E. (2013). The role of fitness in the association between fatness and cardiometabolic risk from childhood to adolescence. Pediatr Diabetes, 14(1):57-65. doi: 10.1111/j.1399-5448.2012.00893.

4. Friedemann, C., Heneghan, C., Mahtani, K., Thompson, M., Perera, R., Ward, A.M. (2012). Cardiovascular disease risk in healthy children and its association with body mass index: systematic review and meta-analysis. BMJ, 345:e4759

5. Barclay, L., Lie, D. (2010). Waist-to-height ratio may predict cardiometabolic risk in normal-weight children CME. BMC Pediatr, 10: 73.

6. Luft, V.M. (2010) Trofolohycheskyy status: kryteryy otsenky y dyahnostyky narushenyy pytanyya [Trophological status: criteria for the evaluation and diagnosis of eating disorders]. SPb., $74 \mathrm{p}$.

7. National health and nutrition examination survey
(2014). Cardiovascular fitness procedures manual. 255 p.

8. Tanaka, H., Monahan, K.D., Seals, D.R. (2001). Agepredicted maximal heart rate revisited. J Am CollCardiol, 37(1):153-6.

9. Glass, S., Gregory, B. (2007). ACSM's Metabolic Calculations Handbook. Lippincott Williams \& Wilkins, Baltimore, 25-74 p.

Received: $\quad$ 13-Oct. - 2016

Accepted: 16-Dec. - 2016 